\title{
Blueberries extract supplementation improves physical performance and decreases oxidative stress in mice
}

\author{
Wei Ping* and Jin Hong-mei \\ Yiwu Industrial and Commercial College, 322000, Yiwu Zhejiang, China. \\ Accepted 29 July, 2011
}

\begin{abstract}
The purpose of the present study was to investigate the effects of blueberry extracts (BBE) supplementation on physical performance and exercise-induced oxidative stress. $\mathbf{4 0}$ male Swiss mice were randomly divided into four groups (three BBE supplementation groups and one control group). The control group was gavaged with distilled water and BBE supplementation groups were gavaged with $\operatorname{BBE}(1,2$ and $4 \mathrm{ml}$ respectively). After 21 days, forced swimming test was performed and the result shows that BBE supplementation could extend the swimming time to exhaustion of the mice, decreasing the blood lactic acid and MDA levels, while increasing SOD, CAT and GPX activities. It was concluded that BBE supplementation improves physical performance (swimming time) and decreases oxidative stress.
\end{abstract}

Key words: Blueberries extract, physical performance, oxidative stress, mice.

\section{INTRODUCTION}

Physical exercise provides many benefits to human health, therefore is recommended for the prevention and management of many chronic diseases and for the maintenance of optimal health (Chang et al., 2007). However, intense physical exercise can increase oxygen consumption by up to 10 - to 20 -fold over resting levels to meet energy demands, and oxygen uptake in active skeletal muscle increase 100- to 200-fold (Sureda et al., 2009). Increased oxygen uptake during exercise is accompanied by an elevation in reactive oxygen species (ROS), and ROS are harmful to cells, mainly because they injure lipids, proteins and nucleic acids, which leads to structural and functional impairments (Duthie et al., 1990; Mantle and Preedy, 1999; Alessio et al., 2000; Jackson, 2005). So, intense physical exercise is generally recognized as a factor inducing an oxidative stress. Given the potential involvement of ROS in detrimental cellular processes, research has focused on the potential beneficial effects of antioxidant consumption. Some studies have indicated that antioxidant supplementations, such as vitamins $C$ and $E$, attenuate

${ }^{*}$ Corresponding author. E-mail: WeiPing75@qq.com. Tel: +860579-83803551. Fax: +86-0579-83803551. oxidative stress and prevent strenuous exercise-induced oxidative injury in human subjects and rats (Goldfarb, 1999; Sacheck et al., 2003; Bloomer et al., 2006).

Many fresh fruits and vegetables have been found to contain natural antioxidants, which provide protection against harmful free radicals and have been associated with a number of health benefits (Padilla et al., 2008; Palmer and Kitchin, 2010). Blueberries are flowering plants that belong to Vaccinium spp. of the family Ericaceae. Several pharmacological activities of blueberry extracts (BBE) have been documented, including ophthalmic activity and anti-aging, anti-cancer, antibacterial, anti-angiogenesis, anti-obesity and anti-diabetic properties (Ahmet et al., 2009; Chen et al., 2010; Gordillo et al., 2009; Kolosova et al., 2004; Vuong et al., 2009). Recent studies have shown that blueberries contain anthocyanins, polyphenols and flavonoids, and appear to have the highest antioxidant capacity among the common fruits and vegetables (Ahmet et al., 2009). Though, BBE has been reported to reduce the formation of the lipid peroxidation and to be an effective antioxidant (Dulebohn et al., 2008), to our knowledge, the effect of BBE supplementation on oxidative stress induced by Intense exercise is still poorly understood. Therefore, the purpose of the present study was to investigate the effects of BBE supplementation on physical performance and exercise- 
induced oxidative stress.

\section{MATERIALS AND METHODS}

\section{Plant material and preparation of blueberry extracts}

Fresh blueberries (Vaccinium corymbosum L) were supplied by Zhejiang Blueberry Biotechnology Co., Ltd. (Huzhou, China). Blueberry extracts (BBE) were prepared, with fresh blueberry (100 g) weighed, mixed with $100 \mathrm{ml}$ of distilled water and then milled using a commercial mini-processor. The crushed berries were put in centrifuge tubes. Tubes were centrifuged (3000 g, $15 \mathrm{~min}$ ) and the clear supernatant fluid was collected and used either within $1 \mathrm{~h}$ of collection or stored at $-80^{\circ} \mathrm{C}$ for further work.

\section{Experimental animals}

Male Swiss mice (20 to $22 \mathrm{~g}$ body weight) were obtained from the Experimental Animal Center of Zhejiang Province (Certificate no. 20061348). The animals were housed under diurnal lighting conditions (12 h /12 h) and allowed free access to food and water. All animal experiments were performed in accordance with the Guide for the Care and Use of Laboratory Animals of the Chinese National Institutes of Health and with the ethical approval of the Zhejiang Medical Laboratory Animal Care and Use Committee as well as the Ethical Committee of Yiwu Industrial and Commercial College.

\section{Experimental protocol}

Animals were allowed to adapt to their surroundings for 1 week before the experiments started. After adaptation, forty mice were randomly divided into four groups with 10 mice each: 1 , C group: control group (the mice were gavaged at 10:00 once a day with 4 $\mathrm{ml}$ of distilled water for 21 consecutive days); 2, BBE-H group: BBE supplementation group at a high dose (the mice were gavaged at 10:00 once a day with $4 \mathrm{ml}$ of BBE for 21 consecutive days); 3 , BBE-M group: BBE supplementation group at a middle dose (the mice were gavaged at 10:00 once a day with $2 \mathrm{ml}$ of $B B E$ and $2 \mathrm{ml}$ of distilled water for 21 consecutive days); 4, BBE-L group: BBE supplementation group at a low dose (the mice were gavaged at 10:00 once a day with $1 \mathrm{ml}$ of BBE and $3 \mathrm{ml}$ of distilled water for 21 consecutive days).

After each supplementation, all groups of the mice were allowed to rest for $30 \mathrm{~min}$ and were forced to swim for $20 \mathrm{~min}$ to become accustomed to swimming. The swimming exercise was employed in the study to evaluate the effects of BBE supplementation on physical performance and exercise-induced oxidative stress.

The swimming exercise was carried out in an adjustable-current water pool $(50 \times 50 \times 40 \mathrm{~cm})$, filled with water to $30 \mathrm{~cm}$ depth and maintained at a temperature of $25 \pm 1^{\circ} \mathrm{C}$. The current in the pool was generated by circulating water with a pump, and the strength of the current was adjusted to $8 \mathrm{~L} / \mathrm{min}$ with a water flow metre (type F45500, Blue White Co., Westminster, CA, USA). The water was agitated to keep the mice limbs moving. After the final treatment with BBE or distilled water, the mice were allowed to rest for $30 \mathrm{~min}$. Then, they were made to swim in groups until exhaustion. Mice were considered to be exhausted when they failed to rise to the surface of the water to breathe within a $7 \mathrm{~s}$ period. The swimming times until exhaustion were used as the index of physical performance.

\section{Biochemical analysis}

At the end of swimming exercise, the mice were sacrificed under light ether anesthesia and whole blood samples were collected in tubes with anticoagulant by heart puncture. Blood samples were centrifuged at $1400 \mathrm{~g}$ and $4^{\circ} \mathrm{C}$ for $10 \mathrm{~min}$. The supernatant fractions (plasma) were used for the determination of the contents of lactic acid (LA). In addition, immediately after the blood had been collected, the skeletal muscle was dissected out quickly, washed with physiological saline and dried with absorbent paper. Then, the content of GPX, CAT, SOD and MDA were analyzed.

LA was determined using a commercial diagnostic kit (Beijing Leadman Biochemistry Technology Co. Ltd., Beijing, China). GPX, CAT, SOD and MDA were determined using commercial diagnostic kits (Institute of Biological Engineering of Nanjing Jianchen, Nanjing, China).

\section{Statistical analysis}

Statistical analysis was carried out using ANOVA followed by posthoc Turkey test (SPSS 15 for Windows). The criterion of significance was set at $P<0.05$. All results were given as mean \pm SD.

\section{RESULTS}

\section{BBE supplementation improves physical performance}

The swimming time until exhaustion of mice was considered as an index of physical performance. As shown in Figure 1, the swimming time until exhaustion of BBE supplementation groups (BBE-L, BBE-M and BBE-H group) was significantly longer than that of the control group $(P<0.05)$, and the swimming times increased by $26.6,42.1$ and $49.7 \%$, respectively.

\section{BBE supplementation decreased blood LA}

As shown in Figure 2, the LA levels of BBE supplementation groups was significantly lower than that of the control group $(P<0.05)$, and the LA levels decreased by $19.8,27.5$ and $30.2 \%$, respectively.

\section{BBE supplementation enhanced antioxidant enzymes activities}

As shown in Table 1, the GPX activities of BBE supplementation groups was significantly higher than that of the control group $(P<0.05)$, and the GPX activities increased by $17.6,26.1$ and $32.1 \%$, respectively. The CAT activities of BBE supplementation groups was significantly higher than that of the control group $(P<0.05)$, and the CAT activities increased by $24.8,29.8$ and $35.4 \%$, respectively. The SOD activities of BBE supplementation groups was significantly higher than that of the control group $(P<0.05)$, and the SOD activities increased by $27.4,58.9$ and $76.4 \%$, respectively.

\section{BBE supplementation decreased MDA}

As shown in Figure 3, the MDA levels of BBE 


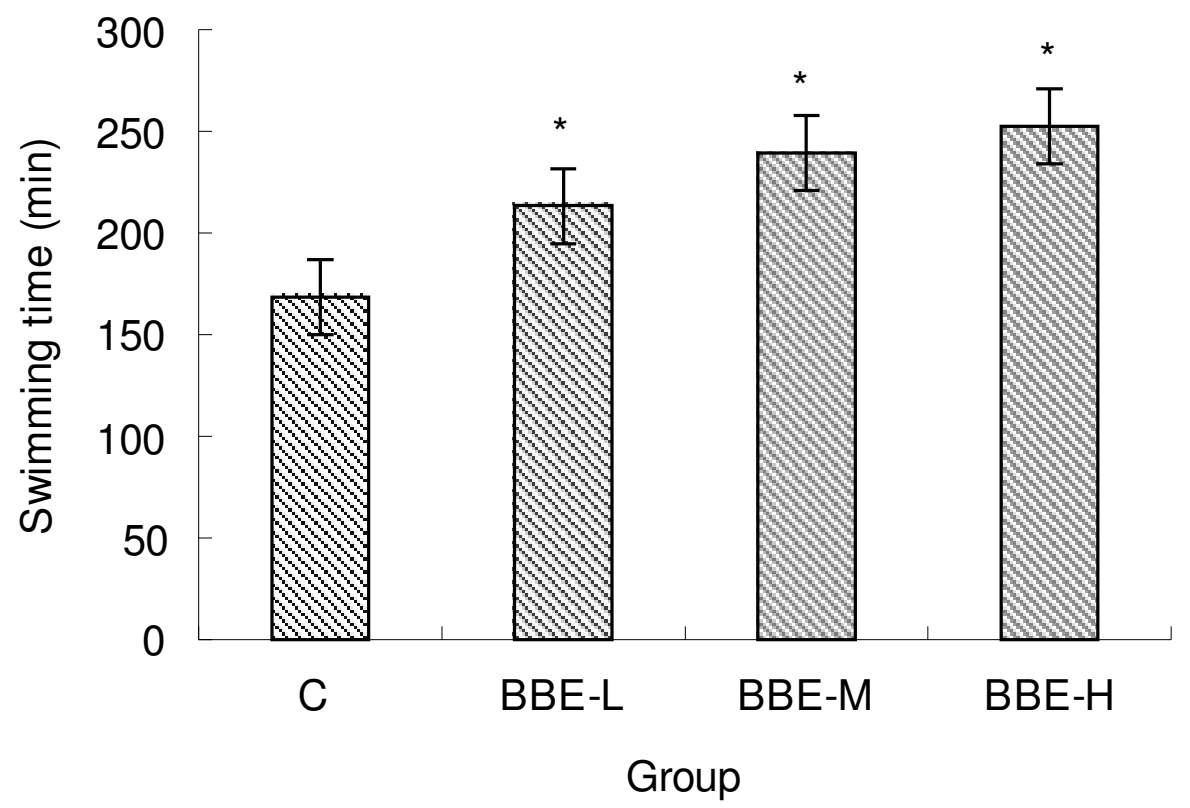

Figure 1. Effect of BBE supplementation on the swimming time until exhaustion of mice. Values are expressed as mean $\pm S D(n=3)$ of 10 mice per group ( $\left.{ }^{*} P<0.05\right)$.

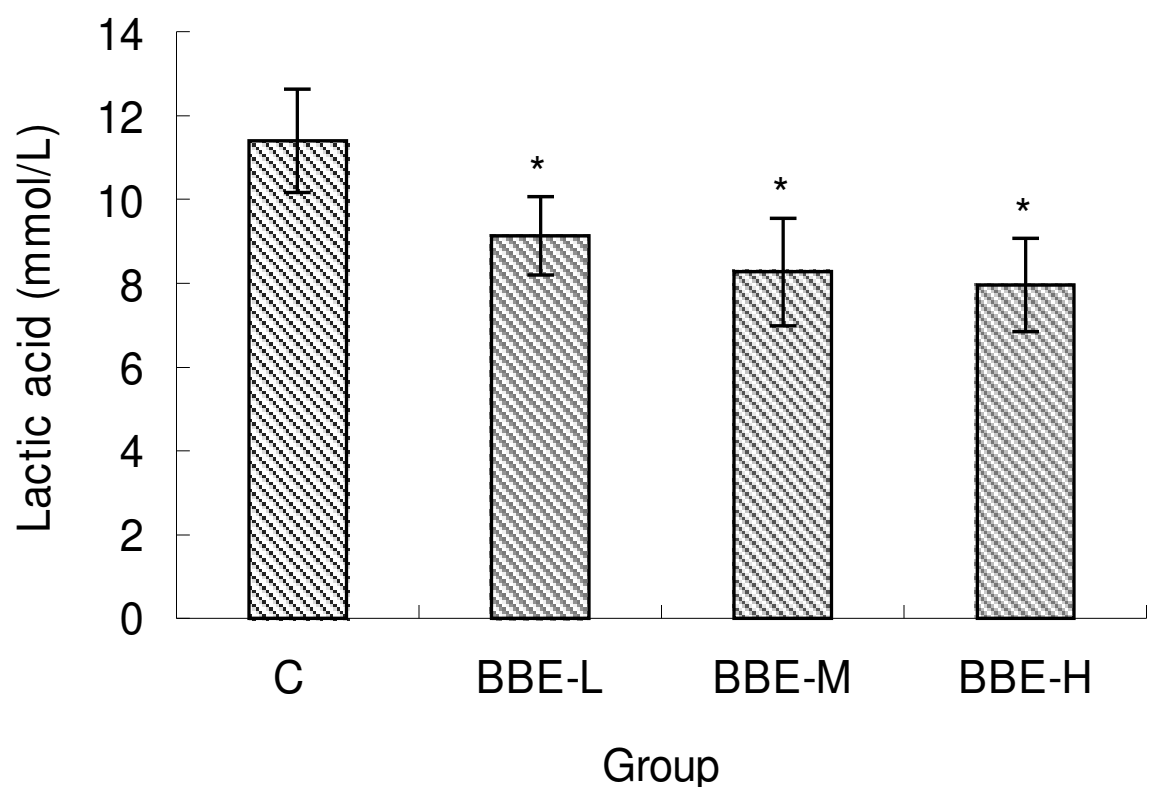

Figure 2. Effect of BBE supplementation on the blood LA of mice. Values are expressed as mean $\pm S D(n=3)$ of 10 mice per group $\left({ }^{*} P<0.05\right)$.

Table 1. Effect of BBE supplementation on the antioxidant enzymes of skeletal muscle of mice.

\begin{tabular}{lccc}
\hline Group & GPX (u/mg.pro) & CAT (u/mg.pro) & SOD (NU/mg.pro) \\
\hline C & $54.28 \pm 3.34$ & $1.41 \pm 0.28$ & $84.27 \pm 7.25$ \\
BBE-L & $63.84 \pm 3.65^{*}$ & $1.76 \pm 0.32^{*}$ & $107.36 \pm 8.41^{*}$ \\
BBE-M & $68.43 \pm 4.11^{*}$ & $1.83 \pm 0.36^{*}$ & $133.87 \pm 6.82^{*}$ \\
BBE-H & $71.69 \pm 2.82^{*}$ & $1.91 \pm 0.41^{*}$ & $149.63 \pm 9.37^{*}$ \\
\hline
\end{tabular}

Values are expressed as mean $\pm S D(n=3)$ of 10 mice per group $\left({ }^{\star} P<0.05\right)$. 


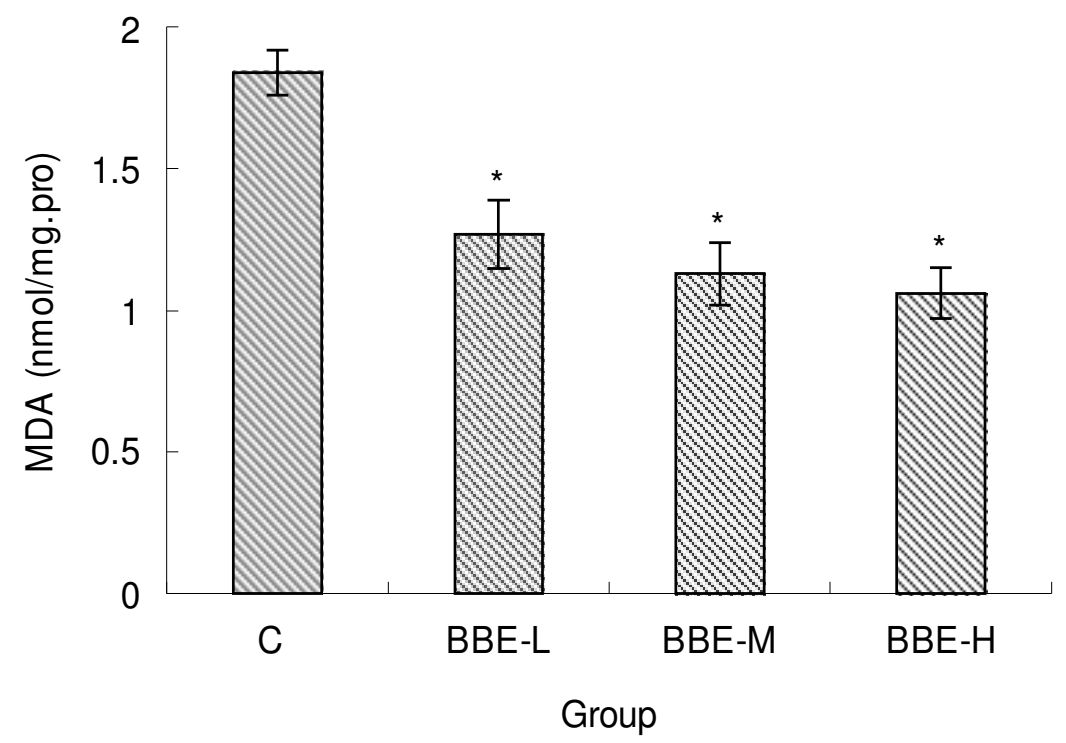

Figure 3. Effect of BBE supplementation on the MDA of skeletal muscle of mice. Values are expressed as mean $\pm S D(n=3)$ of 10 mice per group $\left({ }^{*} P<\right.$ 0.05).

supplementation groups was significantly lower than that of the control group $(P<0.05)$, and the MDA levels decreased by $44.9,62.8$ and $73.6 \%$, respectively.

\section{DISCUSSION}

Supplementation with antioxidants, through an increased consumption in the diet, has become extremely popular as a means to improve one's health or increase physical performance. It has been suggested that increasing the circulating levels of certain antioxidants will help to prevent the accumulation of free radicals inside our cells, thus reducing oxidative stress (McAnulty et al., 2010). Previous studies indicated that blueberry extracts showed antioxidant activity and inhibited lipid peroxidation in vitro (Faria et al., 2005; Dulebohn et al., 2008). In this study, the effects of blueberry extracts supplementation on physical perfor-mance and exercise-induced oxidative stress were investigated.

Swimming, the exhaustive type of exercise, has been selected here as a model of physical exercise, because muscle trauma caused by other types of physical exercise like prolong running in treadmill, exercise stimulated electric shock and plyometric contractions could be avoided (Venditti et al., 1996). In the current study, the swimming time until exhaustion of BBE supplementation groups was significantly longer than that of the control group. The findings demonstrated that BBE supplementation improves physical performance.

The response to exercise in mammals begins with an increase in aerobic muscular activity, which switches over to anaerobic metabolism if the exercise is intense, and leads to the accumulation of lactic acid (LA). With intense exercise, $\mathrm{O}_{2}$ and pyruvic acid are reduced by lactic acid dehydrogenase to lactic acid, which decreases the $\mathrm{pH}$, affecting both the cardio-circulating system and the skeletal muscle system function (Jia and Wu, 2008; LaTorre et al., 2009). In the current study, the LA levels of BBE supplementation groups were significantly lower than that of the control group. Moreover, there is a dosedependent effect, which is another confirmation that BBE supplementation improves physical performance.

Skeletal muscle may be subjected to a greater level of oxidative stress during intense exercise than liver and heart due to increased ROS production. Therefore, the muscle needs greater antioxidant protection against potential oxidative damage occurring during and/or after intense exercise (Ji, 1999; Peake and Suzuki, 2004). SOD, CAT and GPX provide the primary defense against ROS generated during intense exercise. SOD catalyses the conversion of the superoxide radical $\left(\mathrm{O}_{2}{ }^{-}\right)$to $\mathrm{H}_{2} \mathrm{O}_{2}$ and $\mathrm{H}_{2} \mathrm{O}$; CAT then converts $\mathrm{H}_{2} \mathrm{O}_{2}$ to $\mathrm{H}_{2} \mathrm{O}$ and $\mathrm{O}_{2}$; GPX reduces $\mathrm{H}_{2} \mathrm{O}_{2}$ to $\mathrm{H}_{2} \mathrm{O}$ by oxidizing glutathione $(\mathrm{GSH})$. Further, CAT is involved in detoxification of high concentration of $\mathrm{H}_{2} \mathrm{O}_{2}$ and $\mathrm{GPX}$ is sensitive to lower concentration of $\mathrm{H}_{2} \mathrm{O}_{2}$ (Ajmani et al., 2003; Saxena et al., 2010). In the current study, SOD, CAT and GPX activities of BBE supplementation groups was significantly higher than that of the control group. The findings demonstrated that BBE supplementation can promote increase in the activities of these antioxidant enzymes and decrease exercise-induced oxidative stress.

The superoxide radical $\left(\mathrm{O}_{2}{ }^{-}\right)$can combine with iron and form reactive hydroxyl radicals that attack polyunsaturated fatty acids in cell membranes and initiate 
a chain of lipid peroxidation reactions that are the basis for part of the membrane disruption associated with exercise. Lipid peroxidation results in the formation of numerous aldehydes of different chain lengths, such as the 3-carbon product malondialdehyde (MDA), which has been shown to increase with dynamic resistance exercise (Volek et al., 2002). In the current study, the MDA levels of BBE supplementation groups were significantly lower than that of the control group. The results show that BBE supplementation decreased lipid peroxidation, again supporting that BBE has protective effects against exercise-induced oxidative stress.

\section{Conclusion}

From the present findings, we can conclude that blueberries extract supplementation improves physical performance (swimming time) and decreases oxidative stress. The putative mechanism of this effect is that the active ingredients of blueberries contain anthocyanins, polyphenols and flavonoids, and these compounds are antioxidants, which may elevate antioxidant enzymes activities or destroy the free radical generation in the cells. In addition, blueberries extract can delay the increase of lactic acid, which will help increase aerobic and anaerobic exercise capacity. Our data are in reference to mice. Future work using different subjects, possibly of different sporting backgrounds is needed to extend these findings.

\section{ACKNOWLEDGEMENTS}

The authors thank Prof. Zhang Lan (Zhejiang Yuexiu University of Foreign Languages) for the artistic work in the statistical analysis and figure. This work was supported by Yiwu Industrial and Commercial College.

\section{REFERENCES}

Ahmet I, Spangler E, Shukitt-Hale B, Juhaszova M, Sollott SJ, Joseph JA, Ingram DK, Talan M (2009). Blueberry-enriched diet protects rat heart from ischemic damage. PLoS One. 4(6): e5954.

Ajmani RS, Fleg JL, Demehin AA, Wright JG, O'Connor F, Heim JM, Tarien E, Rifkind JM (2003). Oxidative stress and hemorheological changes induced by acute treadmill exercise. Clin. Hemorheol. Microcirc. 28(1): 29-40.

Alessio HM, Hagerman AE, Fulkerson BK, Ambrose J, Rice RE, Wiley $R L$ (2000). Generation of reactive oxygen species after exhaustive aerobic and isometric exercise. Med. Sci. Sports Exerc. 32(9): 15761581.

Bloomer RJ, Goldfarb AH, McKenzie MJ (2006). Oxidative stress response to aerobic exercise: comparison of antioxidant supplements. Med. Sci. Sports Exerc. 38(6): 1098-1103.

Chang WH, Chen CM, Hu SP, Kan NW, Chiu CC, Liu JF (2007). Effect of purple sweet potato leaf consumption on the modulation of the antioxidative status in basketball players during training. Asia. Pac. J. Clin. Nutr. 16(3): 455-461.

Chen CF, Li YD, Xu Z (2010). Chemical principles and bioactivities of blueberry. Yao Xue Xue Bao. 45(4): 422-429.

Dulebohn RV, Yi W, Srivastava A, Akoh CC, Krewer G, Fischer J (2008). Effects of blueberry (Vaccinium ashei) on DNA damage, lipid peroxidation, and phase II enzyme activities in rats. J. Agric. Food Chem. 56(24): 11700-11706.

Duthie GG, Robertson JD, Maughan RJ, Morrice PC (1990). Blood antioxidant status and erythrocyte lipid peroxidation following distance running. Arch. Biochem. Biophys. 282: 78-83.

Faria A, Oliveira J, Neves P, Gameiro P, Santos-Buelga C, de Freitas V, Mateus $N$ (2005). Antioxidant properties of prepared blueberry (Vaccinium myrtillus) extracts. J. Agric. Food Chem. 53(17): 68966902.

Goldfarb AH (1999). Nutritional antioxidants as therapeutic and preventive modalities in exercise-induced muscle damage. Can. J. Appl. Physiol. 24(3): 249-266.

Gordillo G, Fang H, Khanna S, Harper J, Phillips G, Sen CK (2009). Oral administration of blueberry inhibits angiogenic tumor growth and enhances survival of mice with endothelial cell neoplasm. Antioxid. Redox. Signal. 11(1): 47-58.

Jackson MJ (2005). Reactive oxygen species and redox-regulation of skeletal muscle adaptations to exercise. Philos. Trans. R. Soc. Lond. B. Biol. Sci. 360(1464): 2285-2291.

Ji LL (1999). Antioxidants and oxidative stress in exercise. Proc. Soc. Exp. Biol. Med. 222: 283-292.

Jia JM, Wu CF (2008). Antifatigue activity of tissue culture extracts of Saussurea involucrate. Pharm. Biol. 46: 433-436.

Kolosova NG, Lebedev PA, Dikalova AE (2004). Comparison of antioxidants in the ability to prevent cataract in prematurely aging OXYS rats. Bull. Exp. Biol. Med. 137(3): 249-251.

LaTorre A, Crespi D, Serpiello FR, Merati G (2009). Heart rate and blood lactate evaluation in bouldering elite athletes. J. Sports Med. Phys. Fitness, 49(1): 19-24.

Mantle D, Preedy VR (1999). Free radicals as mediators of alcohol toxicity. Adverse Drug React. Toxicol. Rev. 18(4): 235-252.

McAnulty SR, Nieman DC, Fox-Rabinovich M, Duran V, McAnulty LS, Henson DA, Jin F, Landram MJ (2010). Effect of n-3 fatty acids and antioxidants on oxidative stress after exercise. Med. Sci. Sports Exerc. 42(9): 1704-1711.

Padilla FC, Rincón AM, Bou-Rached L (2008). Polyphenol content and antioxidant activity of several seeds and nuts. Arch. Latinoam. Nutr. 58(3): 303-308.

Palmer DM, Kitchin JS (2010). A double-blind, randomized, controlled clinical trial evaluating the efficacy and tolerance of a novel phenolic antioxidant skin care system containing Coffea arabica and concentrated fruit and vegetable extracts. J. Drugs Dermatol. 9(12): 1480-1487.

Peake J, Suzuki K (2004). Neutrophil activation, antioxidant supplements and exercise-induced oxidative stress. Exerc. Immunol. Rev. 10: 129-141.

Sacheck JM, Milbury PE, Cannon JG, Roubenoff R, Blumberg JB (2003). Effect of vitamin $E$ and eccentric exercise on selected biomarkers of oxidative stress in young and elderly men. Free Radic. Biol. Med. 34(12): 1575-1588.

Saxena S, Shukla D, Saxena S, Khan YA, Singh M, Bansal A, Sairam M, Jain SK (2010). Hypoxia preconditioning by cobalt chloride enhances endurance performance and protects skeletal muscles from exercise-induced oxidative damage in rats. Acta. Physiol (Oxf). 200(3): 249-263.

Sureda A, Ferrer MD, Tauler P, Romaguera D, Drobnic F, Pujol P, Tur JA, Pons A (2009). Effects of exercise intensity on lymphocyte $\mathrm{H} 2 \mathrm{O} 2$ production and antioxidant defences in soccer players. Br. J. Sports Med. 43(3): 186-190.

Venditti P, Piro MC, Artiaco G, Di Meo S (1996). Effect of exercise on tissue anti-oxidant capacity and heart electrical properties in male and female rats. Eur. J. Appl. Physiol. Occup. Physiol. 74(4): 322329.

Volek JS, Kraemer WJ, Rubin MR, Gómez AL, Ratamess NA, Gaynor P (2002). L-Carnitine L-tartrate supplementation favorably affects markers of recovery from exercise stress. Am. J. Physiol. Endocrinol. Metab. 282(2): E474-478.

Vuong T, Benhaddou-Andaloussi A, Brault A, Harbilas D, Martineau LC, Vallerand D, Ramassamy C, Matar C, Haddad PS (2009). Antiobesity and antidiabetic effects of biotransformed blueberry juice in $\operatorname{KKA}(\mathrm{y})$ mice. Int. J. Obes (Lond). 33(10): 1166-1173. 